\title{
Cohomological classification of braided Ann-categories
}

\author{
Nguyen Tien Quang • Dang Dinh Hanh
}

Received: 15 December 2011 / Accepted: 15 May 2013 / Published online: 12 June 2013

(C) Tbilisi Centre for Mathematical Sciences 2013

\begin{abstract}
Roughly speaking, a braided Ann-category, or braided categorical ring, $\mathcal{A}=(\mathcal{A}, \oplus, \otimes, \mathbf{c})$ is a groupoid $\mathcal{A}$ carrying the structure of a commutative ring up to some natural isomorphisms satisfying suitable coherence conditions. For any given braided Ann-category $\mathcal{A}$, the set $\pi_{0} \mathcal{A}$ of isomorphism classes of objects in $\mathcal{A}$ is a commutative ring, and the set $\pi_{1} \mathcal{A}$ of automorphisms of the zero object of $\mathcal{A}$ carries a canonical structure of $\pi_{0} \mathcal{A}$-module. As a main result in the paper, we establish a classification theorem for braided Ann-categories $\mathcal{A}$ in terms of the elements of a certain 3 rd cohomology group of the commutative ring $\pi_{0} \mathcal{A}$ with coefficients in $\pi_{1} \mathcal{A}$.
\end{abstract}

Keywords Braided Ann-category - Structure transport - Classification theorem · Cohomology of rings

Mathematics Subject Classification (2000) $\quad 18 \mathrm{D} 10 \cdot 16 \mathrm{E} 40$

\section{Introduction}

The notion of monoidal categories, or tensor categories, was introduced by Mac Lane [17], Bénabou [2] in 1963. Later, these categories were "refined" to become categories with group structure, when the notion of invertible objects was added (see Laplaza [14], Rivano [26]). Now, if the underlying category is a groupoid (i.e., every morphism is an isomorphism), we get the concept of group-like monoidal categories (see Fröhlich

Communicated by Walter Tholen.

N. T. Quang $(\varangle) \cdot$ D. D. Hanh

Department of Mathematics, Hanoi National University of Education, Hanoi, Vietnam

e-mail: cn.nguyenquang@gmail.com

D. D. Hanh

e-mail: ddhanhdhsphn@gmail.com 
and Wall [6]), or Gr-categories (see Sinh [28]), or categorical groups. The manifold of categorical groups has been classified by the cohomology group $H^{3}(G, A)$ (see [28]).

The concept of braided tensor categories introduced by Joyal and Street [10] is a necessary extension of a symmetric tensor category. In [10], the authors have classified the manifold of braided categorical groups by the category of quadratic functions (based on the work of Mac Lane on the representation of quadratic functions by the abelian cohomology group $H_{a b}^{3}(G, A)$ [15]). Previously, the case of symmetric categorical groups (or Picard categories) was solved by Sinh [28].

A generalization of Picard categories was introduced by A. Fröhlich and C. T. C. Wall under the name graded categorical groups [6] (which has been later called graded Picard categories by Cegarra and Khmaladze [5]). Homotopy classification theorems for the manifold of graded categorical groups, the manifold of braided graded categorical groups, and its particular case, the manifold of graded Picard categories have been established in [4,5,7], respectively. Next, Calvo, Cegarra and Quang have homotopy classified braided and symmetric fibred categorical groups by higher cohomology of modules [3]. Each of these categories induces a 3-cocycle in a certain sense, and the equivalent class of this category corresponds to the $3 \mathrm{rd}$ cohomology class.

The categories with two monoidal structures interested many authors. In 1972, Laplaza studied categories with the distributivity constraints [13]. The main result of [13] is a coherence theorem for these categories. Later, in [6], Fröhlich and Wall presented the concept of ring-like categories in order to offer a new list of axioms which is shorter than the one of Laplaza [13]. These two concepts are formalizations of the category of modules over a commutative ring.

In 1994, Kapranov and Voevodsky [12] omitted the requirements of the axiomatics of Laplaza which are related to the commutativity constraints of the operation $\otimes$ and called them ring categories.

Quang introduced a "stricter" concept than that of ring category, called Ann-categories, as a categorification of rings, in which objects and morphisms of the underlying category are required to be invertible, which is analogous to categorical groups (see $[7,18]$ ). When $\mathcal{P}$ is a Picard category, then the category $\operatorname{End}(\mathcal{P})$ of Pic-functors on $\mathcal{P}$ is an Ann-category (see $[9,19])$.

In 2006, Jibladze and Pirashvili [11] presented the concept of categorical rings as a slightly modified version of the concept of Ann-categories. Quang and his coauthors proved in [24] that the manifold of Ann-categories is contained in that of ring categories, and Quang has recently given an example of an Ann-category which is not a categorical ring.

Braided Ann-categories (in particular, symmetric Ann-categories) were considered in [23]. In that paper we showed that the two concepts of "distributive categories" of Laplaza and "ring-like categories" of Fröhlich and Wall coincide, and are broader than that of symmetric Ann-categories.

The purpose of this paper is to classify the braided Ann-categories and to expand known results on Ann-categories. The content of the paper is as follows. In Sect. 2 we recall results of calculations of Mac Lane cohomology groups $H_{M a c L}^{i}(R, M)$, and of Shukla cohomology groups $H_{S h u}^{i}(R, M), i=1,2,3$, of a ring $R$. They are used to classify Ann-categories (see [20]), regular Ann-categories (see [21]). Based 
on our studies of braided Ann-functors in terms of homotopy relation and of Anncategory structures in terms of cohomology relation, we define the groups $H_{a b}^{i}(R, M)$, $i=1,2,3$, of an commutative ring $R$ as cohomology groups of a truncated complex, which is analogous to Sinh's method for Picard categories [28]. We also interpret the groups $H_{a b}^{i}(R, M), i=2,3$, by the extension problem of commutative rings.

In Sect. 3 we present the structure transport of braided Ann-category, which extends the results of Rivano [26], of Sinh [28], and of Quang [20] for monoidal categories, for Gr-categories, and for Ann-categories, respectively. With the structure transport, we construct reduced braided Ann-categories, called braided Ann-categories of type $(R, M)$ in Sect. 4. The homotopy classification of braided Ann-functors is dealt with in Section 5. This result is used to classify braided Ann-categories (Theorem 6.1) in Sect. 6. We also prove a classification theorem of braided Ann-categories having the same first two invariants, that is, they are of the same type $(R, M)$ (Theorem 6.2).

In this paper, we sometimes write $X Y$ instead of $X \otimes Y$ for two objects $X, Y$.

\section{Cohomology for rings}

\subsection{Mac Lane cohomology groups for rings}

Let $R$ be a ring with unit and $M$ be a $R$-bimodule. From the definition of ring cohomology of Mac Lane [16], we may obtain the description of elements of the cohomology group $H_{M a c L}^{3}(R, M)$ (see Proposition 7.2, Proposition 7.3 [20]).

The group $Z_{\text {MacL }}^{3}(R, M)$ of 3-cocycles of the ring $R$ with coefficients in the $R$-bimodule $M$ consists of 5-tuples $(\xi, \eta, \alpha, \lambda, \rho)$ of maps

$$
\xi, \alpha, \lambda, \rho: R^{3} \rightarrow M, \eta: R^{2} \rightarrow M
$$

which satisfy the following conditions for all $x, y, z, t \in R$ :

S1. $\xi(y, z, t)-\xi(x+y, z, t)+\xi(x, y+z, t)-\xi(x, y, z+t)+\xi(x, y, z)=0$,

S2. $\xi(x, y, z)-\xi(x, z, y)+\xi(z, x, y)+\eta(x+y, z)-\eta(x, z)-\eta(y, z)=0$,

S3. $\eta(x, y)+\eta(y, x)=0$,

S4. $x \eta(y, z)-\eta(x y, x z)=\lambda(x, y, z)-\lambda(x, z, y)$,

S5. $\eta(x, y) z-\eta(x z, y z)=\rho(x, y, z)-\rho(y, x, z)$,

S6. $x \xi(y, z, t)-\xi(x y, x z, x t)=\lambda(x, z, t)-\lambda(x, y+z, t)+\lambda(x, y, z+t)$

$-\lambda(x, y, z)$,

S7. $\xi(x, y, z) t-\xi(x t, y t, z t)=\rho(y, z, t)-\rho(x+y, z, t)+\rho(x, y+z, t)$

$-\rho(x, y, z)$,

S8. $\rho(x, y, z+t)-\rho(x, y, z)-\rho(x, y, t)+\lambda(x, z, t)+\lambda(y, z, t)$

$-\lambda(x+y, z, t)=\xi(x z+x t, y z, y t)+\xi(x z, x t, y z)-\eta(x t, y z)$

$$
+\xi(x z+y z, x t, y t)-\xi(x z, y z, x t),
$$

S9. $\alpha(x, y, z+t)-\alpha(x, y, z)-\alpha(x, y, t)=x \lambda(y, z, t)+\lambda(x, y z, y t)$

$-\lambda(x y, z, t)$, 
S10. $\alpha(x, y+z, t)-\alpha(x, y, t)-\alpha(x, z, t)=x \rho(y, z, t)-\rho(x y, x z, t)$

$$
+\lambda(x, y t, z t)-\lambda(x, y, z) t,
$$

S11. $\alpha(x+y, z, t)-\alpha(x, y, t)-\alpha(y, z, t)=-\rho(x, y, z) t-\rho(x z, y z, t)$

$$
+\rho(x, y, z t)
$$

S12. $x \alpha(y, z, t)-\alpha(x y, z, t)+\alpha(x, y z, t)-\alpha(x, y, z t)+\alpha(x, y, z) t=0$,

and satisfy the normalization conditions:

$$
\begin{aligned}
& \xi(0, y, z)=\xi(x, 0, z)=\xi(x, y, 0)=0, \\
& \alpha(1, y, z)=\alpha(x, 1, z)=\alpha(x, y, 1)=0, \\
& \alpha(0, y, z)=\alpha(x, 0, z)=\alpha(x, y, 0)=0, \\
& \lambda(1, y, z)=\lambda(0, y, z)=\lambda(x, 0, z)=\lambda(x, y, 0)=0, \\
& \rho(x, y, 1)=\rho(0, y, z)=\rho(x, 0, z)=\rho(x, y, 0)=0 .
\end{aligned}
$$

The subgroup $B_{M a c L}^{3}(R, M) \subset Z_{M a c L}^{3}(R, M)$ of 3-coboundaries consists of 5-tuples of maps $h=(\xi, \eta, \alpha, \lambda, \rho)$ such that there exist maps $g=(\mu, v): R^{2} \rightarrow M$ satisfying $h=\partial_{M a c L} g$, i.e.,

$$
\begin{aligned}
& \text { S13. } \xi(x, y, z)=\mu(y, z)-\mu(x+y, z)+\mu(x, y+z)-\mu(x, y), \\
& \text { S14. } \quad \eta(y, x)=\mu(x, y)-\mu(y, x), \\
& \text { S15. } \alpha(x, y, z)=x v(y, z)-v(x y, z)+v(x, y z)-v(x, y) z, \\
& \text { S16. } \lambda(x, y, z)=v(x, y+z)-v(x, y)-v(x, z)+x \mu(y, z)-\mu(x y, x z), \\
& \text { S17. } \quad \rho(x, y, z)=v(x+y, z)-v(x, z)-v(y, z)+\mu(x, y) z-\mu(x z, y z),
\end{aligned}
$$

where $\mu, v$ satisfy the normalization condition $\mu(0, y)=\mu(x, 0)=0$ and $v(0, y)=v(x, 0)=v(1, y)=v(x, 1)=0$.

The group $Z_{\text {MacL }}^{2}(R, M)$ consists of 2-cocycles $g=(\mu, v)$ of the ring $R$ with coefficients in the $R$-bimodule $M$ satisfying:

$$
\partial_{M a c L} g=0 .
$$

The subgroup $B_{\text {MacL }}^{2}(R, M) \subset Z_{\text {MacL }}^{2}(R, M)$ of 2-coboundaries are the pairs $(\mu, v)$ such that there exists maps $t: R \rightarrow M$ satisfying $(\mu, v)=\partial_{M a c L} t$, i.e.,

$$
\begin{aligned}
& \text { S18. } \mu(x, y)=t(y)-t(x+y)+t(x), \\
& \text { S19. } v(x, y)=x t(y)-t(x y)+t(x) y,
\end{aligned}
$$

where $t$ satisfies the normalization condition $t(0)=t(1)=0$.

The group $Z_{\text {MacL }}^{1}(R, M)$ consists of 1-cochains $t$, of the ring $R$ with coefficients in the $R$-bimodule $M$, satisfying:

$$
\partial_{M a c L} t=0 .
$$


The subgroup $B_{M a c L}^{1}(R, M) \subset Z_{\text {MacL }}^{1}(R, M)$ consists of 1-coboundaries which are maps $t$ such that there exists $a \in R$ satisfying $t(x)=a x-x a$.

The quotient groups

$$
H_{M a c L}^{i}(R, M)=Z_{M a c L}^{i}(R, M) / B_{M a c L}^{i}(R, M), \quad i=1,2,3
$$

are called the ith Mac Lane cohomology group of the ring $R$ with coefficients in the $R$-bimodule $M$.

\subsection{The low-dimensional cohomology groups of a comutative ring}

To classify braided Ann-categories, we define a truncated cochain complex for the commutative ring $R$ and $R$-module $M$, similar to the work of Sinh on Picard categories in [28]. This complex is defined as follows:

$$
C_{a b}(R, M): 0 \longrightarrow C_{a b}^{1}(R, M) \stackrel{\partial}{\longrightarrow} C_{a b}^{2}(R, M) \stackrel{\partial}{\longrightarrow} Z_{a b}^{3}(R, M) \longrightarrow 0
$$

in which $C_{a b}^{1}(R, M)$ consists of normalized maps $t: R \rightarrow M, C_{a b}^{2}(R, M)$ consists of all pairs $(\mu, v)$ of normalized maps $\mu, v: R^{2} \rightarrow M$ and $Z_{a b}^{3}(R, M)$ consists of all pairs $(h, \beta)$, where $h \in Z_{\text {MacL }}^{3}(R, M)$ and the map $\beta: R \rightarrow M$, satisfying the following 3-cocycle conditions:

S20. $\alpha(x, y, z)-\alpha(x, z, y)+\alpha(z, x, y)+x \beta(y, z)-\beta(x y, z)+y \beta(x, z)=0$,

S21. $\alpha(x, y, z)-\alpha(y, x, z)+\alpha(y, z, x)-y \beta(x, z)+\beta(x, y z)-z \beta(x, y)=0$,

S22. $\beta(x, y)-\beta(x, y+z)+\beta(x, z)=\rho(y, z, x)-\lambda(x, y, z)$.

for $x, y, z \in R$. For each $t \in C_{a b}^{1}(R, M)$, the coboundary $\partial t$ is given by

$$
\partial t=\partial_{M a c L} t
$$

and for each $g \in C_{a b}^{2}(R, M), \partial g$ is given by

$$
\partial g=\left(\partial_{M a c L} g, \beta\right),
$$

where $\beta(x, y)=v(x, y)-v(y, x)$.

Clearly, $H_{a b}^{1}(R, M)=H_{M a c L}^{1}(R, M)$.

Note. Assume that $(h, \beta) \in Z_{a b}^{3}(R, M)$. If the map $\eta$ of the Mac Lane 3-cocycle $h=(\xi, \eta, \alpha, \lambda, \rho)$ satisfies the regular condition

S3b. $\eta(x, x)=0$

for all $x \in R$, then $h$ is a Shukla 3-cocycle [27]. The group of all $(h, \beta)$ with $\eta$ satisfying $\mathrm{S} 3 \mathrm{~b}$ is denoted by $Z_{\text {Shab }}^{3}(R, M)$. 
A full explanation for the groups $H_{a b}^{2}(R, M), H_{S h a b}^{3}(R, M)$ is given in Sect. 6. Now we give an explanation for the group of $H_{a b}^{2}(R, M)$ via extensions of singular commutative ring, and one in part for the group $H_{S h a b}^{3}(R, M)$ based on obstruction theory of extensions of commutative rings.

$2.3 \mathrm{H}_{a b}^{2}$ and extensions of commutative rings

Let $\mathcal{E}$ be the ring extension

$$
\mathcal{E}: 0 \rightarrow A \stackrel{i}{\longrightarrow} S \stackrel{p}{\longrightarrow} R \rightarrow 0
$$

where $R, S$ are rings with units, $p\left(1_{S}\right)=1_{R}$ and $A^{2}=0$. Each $u \in S$ gives an inner bimultiplication $\tau_{u}$ on $S$ by

$$
\tau_{u}(s)=u s,(s) \tau_{u}=s u, s \in S .
$$

Since $A$ is a two-sided ideal in $S$, then the assignment to each $\tau_{u}$ yields a homomorphism

$$
\tau: S \rightarrow M_{A},
$$

where $M_{A}$ is the ring of bimultiplications on $A$ (see [16]). Let $\{u(x) \mid x \in R\}$ be a set of representatives of $R$ in $S$ with $u(0)=0, u(1)=1$. Since $\tau i=0$, then $\tau$ induces a homomorphism

$$
\varphi: R \rightarrow M_{A}, \quad \varphi(x)=\tau_{u(x)}
$$

Then, $\varphi$ induces a structure of $R$-bimodule on $A$. Let

$$
\operatorname{Opext}(A, R, \varphi)
$$

be the set of isomorphism classes of extensions of $A$ by $R$, which induces $\varphi$. We have the following result.

Theorem 2.1 Let $A$ be a singular ring $\left(A^{2}=0\right), R$ a commutative ring with unit and $\varphi: R \rightarrow M_{A}$ a ring homomorphism. Then, there is a bijection

$$
H_{a b}^{2}(R, A) \rightarrow \operatorname{Opext}(R, A, \varphi)
$$

Proof Step 1. For each 2-cocycle $(f, g) \in Z_{a b}^{2}(R, A)$, we define an extension

$$
\mathcal{E}_{(f, g)}: 0 \rightarrow A \stackrel{i}{\longrightarrow} E_{(f, g)} \stackrel{p}{\longrightarrow} R \rightarrow 0
$$

as follows:

$E_{(f, g)}$ is the commutative ring whose underlying set is $A \times R$ and whose operations are given by 


$$
\begin{aligned}
(a, x)+(b, y) & =(a+b+f(x, y), x+y), \\
(a, x) .(b, y) & =(x b+y a+g(x, y), x y) .
\end{aligned}
$$

The associativity, the commutativity of the addition and of the multiplication, and the distributivity of $E_{(f, g)}$ follow from the condition on $(f, g)$. The zero element is $(0,0)$, the opposite of the element $(a, x)$ is

$$
-(a, x)=(-a-f(x,-x),-x)
$$

The homomorphisms $i, p$ are $i(a)=(a, 0), p(a, x)=x$.

Step 2. Each extension $\mathcal{E} \in \operatorname{Opext}(R, A, \varphi)$ corresponds to an extension $\mathcal{E}_{(f, g)}$.

The representation $u(x)$ of the extension (1) induces the functions $f, g: R^{2} \rightarrow A$ given by

$$
\begin{aligned}
& f(x, y)=u(x)+u(y)-u(x+y), \\
& g(x, y)=u(x) u(y)-u(x y) .
\end{aligned}
$$

By $u(0)=u(1)=0$, we deduce $(f, g)$ are normalized.

Checking the axioms of ring on the representation $u(x), x \in R$, we obtain the following relations

$$
\begin{array}{r}
f(y, z)-f(x+y, z)+f(x, y+z)-f(x, y)=0, \\
f(x, y)-f(y, x)=0, \\
x g(y, z)-g(x y, z)+g(x, y z)+z g(x, y)=0, \\
g(x, y)-g(y, x)=0, \\
x f(y, z)-f(x y, x z)+g(x, y+z)-g(x, y)-g(x, z)=0 .
\end{array}
$$

Therefore $(f, g) \in Z_{a b}^{2}(R, A)$.

Since each element in $S$ can be represented uniquely as $a+u(x)$, we have the map

$$
\Phi: S \rightarrow E_{(f, g)}, \quad a+u(x) \mapsto(a, x)
$$

From the 2-cocycle condition of the pair $(f, g)$, it is easy to show that $\Phi$ is a ring isomorphism. Moreover, $\varphi$ induces an equivalence between the extensions $\mathcal{E}$ and $\mathcal{E}_{(f, g)}$.

Step 3. $E_{(f, g)} \cong E_{\left(f^{\prime}, g^{\prime}\right)}$ iff $(f, g)$ and $\left(f^{\prime}, g^{\prime}\right)$ are cohomologous.

Assume that $\Phi: E_{(f, g)} \rightarrow E_{\left(f^{\prime}, g^{\prime}\right)}$ is an isomorphism such that the two extensions $\mathcal{E}_{(f, g)}$ and $\mathcal{E}_{\left(f^{\prime}, g^{\prime}\right)}$ are equivalent. It is obvious that

$$
\Phi(a, x)=(a+t(x), x)
$$

for some map $t: R \rightarrow A$.

From the equations

$$
\begin{gathered}
\Phi[(0, x)+(0, y)]=\Phi(0, x)+\Phi(0, y), \\
\Phi[(0, x) .(0, y)]=\Phi(0, x) . \Phi(0, y),
\end{gathered}
$$


we have

$$
\begin{gathered}
f(x, y)-f^{\prime}(x, y)=t(x)-t(x+y)+t(y), \\
g(x, y)-g^{\prime}(x, y)=x t(y)-t(x y)+y t(x) .
\end{gathered}
$$

This means

$$
(f, g)-\left(f^{\prime}, g^{\prime}\right)=\partial_{a b} t
$$

Conversely, if $(f, g)-\left(f^{\prime}, g^{\prime}\right)=\partial_{a b} t$, by repeating the steps of the above arguments, we have $\mathcal{E}_{(f, g)} \cong \mathcal{E}_{\left(f^{\prime}, g^{\prime}\right)}$.

Corollary 2.2 There exists an isomorphism

$$
H_{a b}^{2}(R, A) \cong D^{1}(R / \mathbb{Z}, A)
$$

in which the right hand side is the Quillen cohomology group of the commutative ring $R$.

Proof This follow from the fomula

$$
D^{1}(R / \mathbb{Z})=\operatorname{Opext}(R, A, \varphi)
$$

(see (4.3) in [25]) and Theorem 2.1.

$2.4 H_{a b}^{3}$ and the obstruction of extensions of commutative rings

Considering the extension of commutative rings

$$
\mathcal{E}: 0 \rightarrow A \stackrel{i}{\longrightarrow} S \stackrel{p}{\longrightarrow} R \rightarrow 0
$$

where $A$ is not necessarily singular. Then, the homomorphism $\tau: S \rightarrow M_{A}$ defines one

$$
\theta: R \rightarrow P_{A}, \quad \theta_{x}=\tau_{u(x)}+\tau(A)
$$

where $P_{A}=M_{A} / \tau(A)$ is the ring of outer bimultiplications of the ring $A$. Clearly, $\theta(1)=1$ and for $\sigma \in \theta_{x}, \sigma^{\prime} \in \theta_{x^{\prime}}$,

$$
\sigma\left(a \sigma^{\prime}\right)=(\sigma a) \sigma^{\prime}, \sigma^{\prime}(a \sigma)=\left(\sigma^{\prime} a\right) \sigma, a \in A \text {. }
$$

One can say that $\theta$ is a regular homomorphism. Moreover, it is strong in the sense that

$$
\sigma \sigma^{\prime}=\sigma^{\prime} \sigma
$$

Now let $A$ be a commutative ring, $R$ a commutative ring with unit, and a strong regular homomorphism $\theta: R \rightarrow P_{A}$. For each $x \in R$, choose $\varphi(x) \in$ 
$\theta x$ such that $\varphi(0)=0, \varphi(1)=i d_{A}$. Then $\varphi$ induces $f, g: R^{2} \rightarrow A$ satisfying

$$
\begin{aligned}
\tau_{f(x, y)} & =\varphi(x)+\varphi(y)-\varphi(x+y), \\
\tau_{g(x, y)} & =\varphi(x) \varphi(y)-\varphi(x y) .
\end{aligned}
$$

The kernel $C_{A}$ of the homomorphism $\tau: A \rightarrow M_{A}$ is called bicenter of $A$. Then, $C_{A}$ consists of elements $c \in A$ with $c a=0=a c$ for all $a \in A$. According to [16] and [20], the structure of the ring $M_{A}$ induces a 3-cocycle $h \in Z_{M a c L}^{3}\left(R, C_{A}\right)$, where $h=(\xi, \eta, \alpha, \lambda, \rho)$ is defined by the following equations:

$$
\begin{aligned}
\xi(x, y, z) & =f(y, z)-f(x+y, z)+f(x+y, z)-f(x, y), \\
\eta(x, y) & =f(x, y)-f(y, x), \\
\alpha(x, y, z) & =x g(y, z)-g(x y, z)+g(x, y z)-z g(x, y), \\
\lambda(x, y, z) & =x f(y, z)-f(x y, x z)+g(x, y+z)-g(x, y)-g(x, z), \\
\rho(x, y, z) & =f(x, y) z-f(x z, y z)+g(x+y, z)-g(x, z)-g(y, z) .
\end{aligned}
$$

The second equation shows that $\eta(x, x)=0$ for all $x \in R$.

On the other hand, $\theta$ is a strong regular homomorphism, so that the map

$$
\beta(x, y)=g(x, y)-g(y, x),
$$

has coefficients in $C_{A}$. We can verify that $(h, \beta)$ satisfies the equations $(S 20)-(S 22)$, so that $k=(h, \beta) \in Z_{\text {Shab }}^{3}\left(R, C_{A}\right)$, such a $k$ is called $a$ obstruction of the strong regular homomorphism $\theta: R \rightarrow P_{A}$.

Note that, the obstruction $(h, \beta)$ is of the form $\delta(f, g)$ but it is not a 3-coboundary since the maps $f, g$ are not in $C_{A}$.

\section{Lemma 2.3 The two obstructions of $\theta$ are cohomologous.}

Proof The definition of the obstruction $(h, \beta)$ depends on the choices of the maps $\varphi, f, g$. According to the proof of Theorem 4 [16], if we change the choice of the map $\varphi$, we can choose $f^{\prime}, g^{\prime}$ such that $h$ is unchanged. It is easy to see that, in this proof, the map $\beta$ is also unchanged.

Now, we fix $\varphi$. If $f^{\prime}, g^{\prime}$ are the maps satisfying (4) and (5), then

$$
\tau_{f(x, y)}=\tau_{f^{\prime}(x, y)}, \quad \tau_{g(x, y)}=\tau_{g^{\prime}(x, y)}
$$

so $f^{\prime}-f=\mu, g^{\prime}-g=v$ are maps with coefficients in $C_{A}$. According to the above proof

$$
h^{\prime}=h+\partial_{M a c L}(\mu, v) \text {. }
$$

Then, the map $\beta^{\prime}$ is defined by

$$
\beta^{\prime}(x, y)=g^{\prime}(x, y)-g^{\prime}(y, x)=\beta(x, y)+v(x, y)-v(y, x) .
$$


This implies

$$
\left(h^{\prime}, \beta^{\prime}\right)=(h, \beta)+\partial_{a b}(\mu, v) .
$$

Proposition 2.4 The strong regular homomorphism $\theta: R \rightarrow P_{A}$ can be realized by a ring extension iff the obstruction $[k]$ of $\theta$ is vanishes in $H_{S h a b}^{3}\left(R, C_{A}\right)$.

Proof Assume that there exists an extension (2) of commutative rings, which induces the strong regular homomorphism $\theta: R \rightarrow P_{A}$. For each $x \in R$, choose a representation $u(x) \in S$ such that $u(0)=0, u(1)=1$. From the definition of obstruction of $\theta$ and by (3), we thus can choose

$$
\begin{aligned}
\varphi(x) & =\tau_{u(x)}, \\
f(x, y) & =u(x)+u(y)-u(x+y), \\
g(x, y) & =u(x) u(y)-u(x y) .
\end{aligned}
$$

With these maps $\varphi, f, g$, we have $k=(h, \beta)$ is identically zero.

Conversely, suppose that $\theta$ has an obstruction $(h, \beta)=\partial_{a b}(\mu, v)$. Then $h=$ $\partial_{M a c L} \mu$ and by Theorem 4 [16], we can choose $(f, g)$ such that $h=0$. Then $\beta=0$.

Now, the set $S=A \times R$ together with two operations given by

$$
\begin{aligned}
(a, x)+(b, y) & =(a+b+f(x, y), x+y), \\
(a, x) .(b, y) & =(a b+\varphi(y) a+\varphi(x) b+g(x, y), x y) .
\end{aligned}
$$

is a commutative ring.

Note. The question is whether there is a realization of an obstruction. That means, given a commutative ring $R$ with unit, a $R$-module $K$ and an element $k \in H_{\text {Shab }}^{3}(R, K)$, does there exist a commutative ring $A$ with bicenter $K$ and a strong regular homomorphism $\theta: R \rightarrow P_{A}$ inducing the obstruction $k$ ?

The above question is equivalent to one of the following questions:

1. Is the concept of above obstruction equivalent to the concept of obstruction due to Barr [1] or not?

2. Does there exist an isomorphism

$$
H_{S h a b}^{3}(R, K) \cong D^{2}(R, K) ?
$$

\section{Braided Ann-categories}

\subsection{Definitions and Examples}

First, let us recall the notion of braided monoidal categories [10]. in $\mathcal{V}$

$A$ braiding of a monoidal category $\mathcal{V}$ consists of a family of natural isomorphisms

$$
c=c_{A, B}: A \otimes B \stackrel{\sim}{\longrightarrow} B \otimes A
$$


such that two diagrams (B1) and (B2) commute:

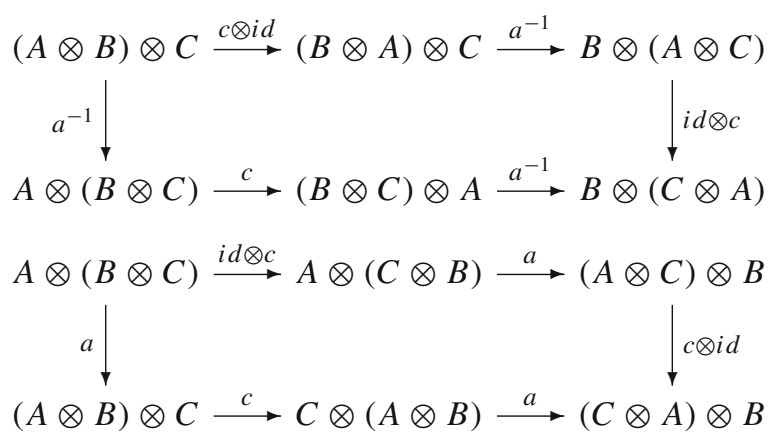

If $c$ is a braiding, so is $c^{\prime}$ given by $c_{A, B}^{\prime}=\left(c_{B, A}\right)^{-1}$, since (B2) is just obtained from (B1) by replacing $c$ with $c^{\prime}$. A symmetry is a braiding $c$ satisfying $c^{\prime}=c$.

A braided monoidal category is a pair $(\mathcal{V}, c)$ consisting of a monoidal category $\mathcal{V}$ and a braiding $c$.

Definition 3.1 [19] An Ann-category consists of:

(i) A category $\mathcal{A}$ together with two bifunctors $\oplus, \otimes: \mathcal{A} \times \mathcal{A} \longrightarrow \mathcal{A}$.

(ii) A fixed object $0 \in \mathcal{A}$ together with natural constraints $a^{+}, c^{+}, g, d$ such that $\left(\mathcal{A}, \oplus, a^{+}, c^{+},(0, g, d)\right)$ is a symmetric categorical group.

(iii) A fixed object $1 \in \mathcal{A}$ together with natural constraints $a, l, r$ such that $(\mathcal{A}, \otimes, a,(1, l, r))$ is a monoidal category.

(iv) Natural isomorphisms $\mathfrak{L}, \mathfrak{R}$

$$
\begin{aligned}
& \mathfrak{L}_{A, X, Y}: A \otimes(X \oplus Y) \rightarrow(A \otimes X) \oplus(A \otimes Y), \\
& \mathfrak{R}_{X, Y, A}:(X \oplus Y) \otimes A \rightarrow(X \otimes A) \oplus(Y \otimes A),
\end{aligned}
$$

such that the following conditions are satisfied:

(Ann-1) For each object $A \in \mathcal{A}$, the pairs $\left(L^{A}, \breve{L^{A}}\right),\left(R^{A}, \breve{R^{A}}\right)$ defined by the relations:

$$
\begin{aligned}
L^{A} & =A \otimes-, & & R^{A}=-\otimes A, \\
\breve{L}_{X, Y}^{A} & =\mathfrak{L}_{A, X, Y}, & & \breve{R}_{X, Y}^{A}=\mathfrak{R}_{X, Y, A}
\end{aligned}
$$

are $\oplus$-functors which are compatible with $a^{+}$and $c^{+}$.

(Ann-2) For all objects $A, B, X, Y \in \mathcal{A}$, the following diagrams commute:

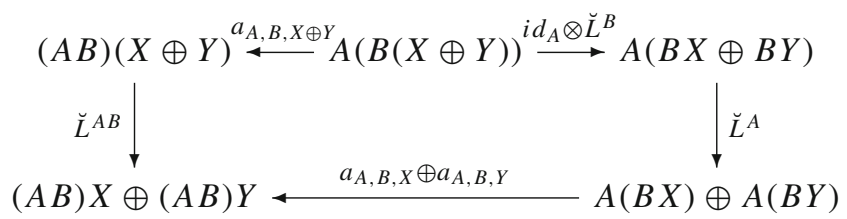



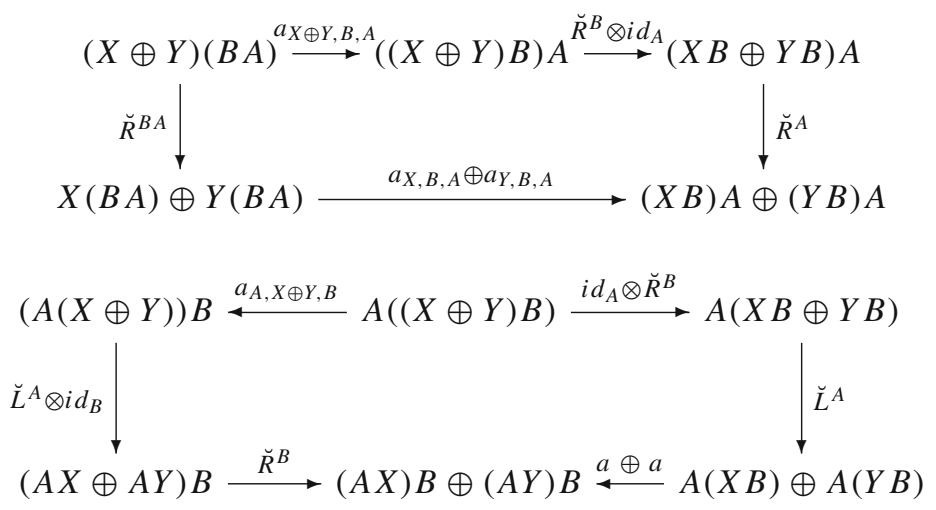

$(A \oplus B) X \oplus(A \oplus B) Y \stackrel{\breve{L}^{A \oplus B}}{\longleftarrow}(A \oplus B)(X \oplus Y) \stackrel{\breve{R}^{X \oplus Y}}{\longrightarrow} A(X \oplus Y) \oplus B(X \oplus Y)$

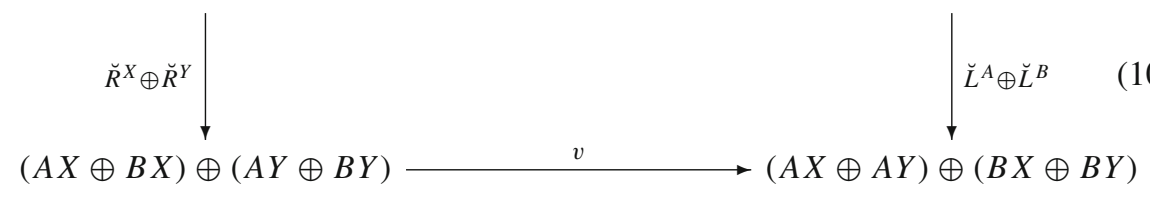

where $v=v_{U, V, Z, T}:(U \oplus V) \oplus(Z \oplus T) \rightarrow(U \oplus Z) \oplus(V \oplus T)$ is the unique morphism built from $a^{+}, c^{+}, i d$ in the symmetric categorical group $(\mathcal{A}, \oplus)$.

(Ann-3) For the unit object $1 \in \mathcal{A}$ of the operation $\otimes$, the following diagrams:

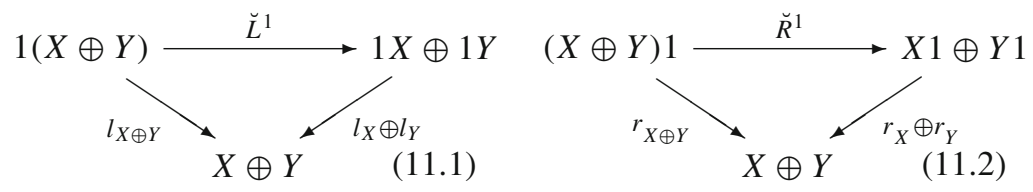

commute.

Definition 3.2 A braided Ann-category $\mathcal{A}$ is an $A n n$-category $\mathcal{A}$ together with a braiding $c$ such that $(\mathcal{A}, \otimes, a, c,(1, l, r))$ is a braided monoidal category, and $c$ makes the following diagram

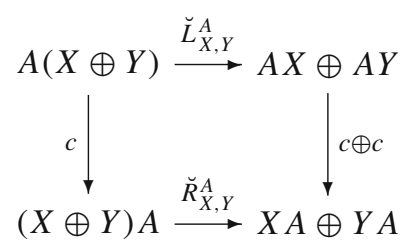

commute and satisfies $c_{0,0}=i d$.

A braided Ann-category is called a symmetric Ann-category if the braiding $c$ is symmetric. A braided Ann-category is called regular if the commutativity constraint $c^{+}$satisfies the condition $c_{X, X}^{+}=i d$ for all $X \in \mathrm{Ob}(\mathcal{A})$. 


\section{Example 3.3 The braided Ann-category of type $(R, M)$}

Let $R$ be a commutative ring with unit and $M$ be an $R$-module. For any normalized 3-cocycle $h \in Z_{\text {MacL }}^{3}(R, M)$, there is an Ann-category $\mathcal{S}=(R, M)$ defined as follows: objects of $\mathcal{S}$ are the elements of $R$, morphisms are automorphisms $(x, a)$ : $x \rightarrow x$ with $a \in M$. The composition law of morphisms in $\mathcal{S}$ is the addition operation in $M$. Two operations $\oplus$ and $\otimes$ of $\mathcal{S}$ are defined by:

$$
\begin{array}{r}
x \oplus y=x+y,(x, a) \oplus(y, b)=(x+y, a+b) ; \\
x \otimes y=x y, \quad(x, a) \otimes(y, b)=(x y, x b+y a) .
\end{array}
$$

The unit constraints of two operations are strict, the associativity and commutativity constraints of $\oplus$, the associativity constraint of $\otimes$ and distributivity constraints are the maps, respectively, of 3-cocycle $h=(\xi, \eta, \alpha, \lambda, \rho)$. If $(h, \beta) \in Z_{a b}^{3}(R, M)$, then $\mathcal{S}$ becomes a braided $A n n$-category with braiding given by the map $\beta: R^{2} \rightarrow M$. Moreover, if $\beta$ satisfying equations

$$
\text { S21b. } \beta(x, y)+\beta(y, x)=0
$$

instead of condition $S 21$, then $\mathcal{S}$ is a symmetric Ann-category.

\section{Example 3.4 The center of an almost strict Ann-category}

Let $\mathcal{A}$ be an almost strict Ann-category, that is, its constraints, except for a distributivity one (left or right) and the symmetry one, are all identities. The center of an Anncategory $\mathcal{A}, C_{\mathcal{A}}$, is a category in which objects are pairs $\left(A, u_{A}\right)$, with $A \in O b(\mathcal{A})$ and

$$
u_{A}=\left(u_{A, X}: A \otimes X \longrightarrow X \otimes A\right)
$$

is a natural transformation satisfying the following three conditions:

$$
\begin{aligned}
u_{1, X} & =i d_{X}, \\
u_{A, X \otimes Y} & =\left(i d_{X} \otimes u_{A, Y}\right) \circ\left(u_{A, X} \otimes i d_{Y}\right), \\
u_{A, X \oplus Y} & =\left(u_{A, X} \oplus u_{A, Y}\right) \circ \breve{L}_{A, X, Y} .
\end{aligned}
$$

A morphism $f:\left(A, u_{A}\right) \rightarrow\left(B, u_{B}\right)$ in $C_{\mathcal{A}}$ is a morphism $f: A \rightarrow B$ in $\mathcal{A}$ satisfying the condition

$$
u_{B, X} \circ(f \otimes i d)=(i d \otimes f) \circ u_{A, Y} .
$$

Then $C_{\mathcal{A}}$ becomes a braided $A n n$-category where the tensor sum and the tensor product of two objects are defined as follows (for details, see [23])

$$
\begin{gathered}
\left(A, u_{A}\right)+\left(B, u_{B}\right)=\left(A \oplus B, \breve{L}_{-, A, B}^{-1} \circ u_{A \oplus B}\right), \\
\left(A, u_{A}\right) \times\left(B, u_{B}\right)=\left(A \otimes B,\left(u_{A} \otimes i d\right) \otimes\left(i d \otimes u_{B}\right)\right) ;
\end{gathered}
$$


the sum and the product of two morphisms in $C_{\mathcal{A}}$ are, respectively, the tensor sum and the tensor product of two morphisms in $\mathcal{A}$; and the braiding $c$ is

$$
c_{\left(A, u_{A}\right),\left(B, u_{B}\right)}=u_{A, B}:\left(A, u_{A}\right) \otimes\left(B, u_{B}\right) \rightarrow\left(B, u_{B}\right) \otimes\left(A, u_{A}\right) .
$$

\section{Example 3.5 The symmetric Ann-category of a strong regular homomorphism}

Let $A, R$ be commutative rings, where $R$ has the unit, and the strong regular homomorphism $\theta: R \rightarrow P_{A}$ (see subsection 2.4). Then, $\theta$ induces the obstruction $k=(h, \beta) \in Z_{\text {Shab }}^{3}\left(R, C_{A}\right)$. Since $h \in Z_{S h u}^{3}\left(R, C_{A}\right)$, then according to the Proposition 6.1 [21], $\left(R, C_{A}, h\right)$ is an Ann-category. Since $\beta$ satisfies conditions (S20), (S21b) and $(\mathrm{S} 22)$, then $\left(R, C_{A}, k\right)$ is a symmetric Ann-category.

In the next sections, we will translate some results on Ann-categories to braided Ann-categories. First, we extend the technique of structure transport to construct the reduced braided $A n n$-category $s \mathcal{A}$ of the braided $A n n$-category $\mathcal{A}$. The category $s \mathcal{A}$ is equivalent to the $A n n$-category $\mathcal{A}$, so we can replace the classification of braided Anncategories by that of their reductions. Diagrams will be used instead of transformations of equations to make it convenient for readers.

\subsection{Braided Ann-functors and the structure transport}

It is known that if $(F, \breve{F}): \mathcal{A} \rightarrow \mathcal{A}^{\prime}$ is a $\oplus$-functor compatible with the associativity constraints of two categorical groups, then it is also compatible with the unit constraints, i.e., one can deduce the isomophism $F_{+}: F(0) \rightarrow 0$ such that $\left(F, \breve{F}, F_{+}\right)$is a monoidal functor. We also recall that, the concept of symmetric monoidal functors is also the concept of braided monoidal functors. With these notes, we have the following definition.

Definition 3.6 Let $\mathcal{A}, \mathcal{A}^{\prime}$ be braided Ann-categories. A braided Ann-functor is an Ann-functor which is compatible with the braidings. In other words, a braided Annfunctor from $\mathcal{A}$ to $\mathcal{A}^{\prime}$ is a quadruple $\left(F, \breve{F}, \widetilde{F}, F_{*}\right)$, where $(F, \breve{F}),\left(F, \widetilde{F}, F_{*}\right)$ are symmetric monoidal functors, respectively, for the operations $\oplus, \otimes$, and satisfies the two following commutative diagrams:

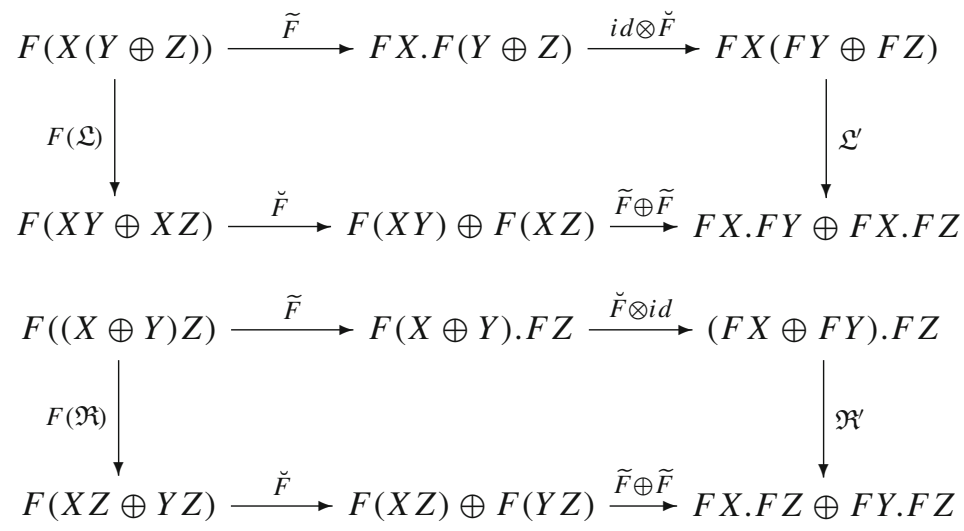


A braided Ann-morphism (or a homotopy)

$$
u:\left(F, \breve{F}, \widetilde{F}, F_{*}\right) \rightarrow\left(K, \breve{K}, \widetilde{K}, K_{*}\right)
$$

of braided $A n n$-functors is a $\oplus$-morphism as well as a $\otimes$-morphism, that is, a natural transformation $u: F \rightarrow K$ monoidal with respect to $\oplus$ and $\otimes$.

If there exists a braided $A n n$-functor $\left(K, \breve{K}, \widetilde{K}, K_{*}\right): \mathcal{A}^{\prime} \rightarrow \mathcal{A}$ and braided Annmorphisms $K F \stackrel{\sim}{\rightarrow} i d_{\mathcal{A}}, F K \stackrel{\sim}{\rightarrow} i d_{\mathcal{A}^{\prime}}$, we say that $\left(F, \breve{F}, \widetilde{F}, F_{*}\right)$ is a braided Annequivalence, and, $\mathcal{A}$ and $\mathcal{A}^{\prime}$ are braided Ann-equivalent.

Adapting classical results on monoidal functors, one can prove that a braided Annfunctor is a braided Ann-equivalence iff $F$ is a categorical equivalence.

We will often use the following elementary fact:

Lemma 3.7 Any braided Ann-functor $F=\left(F, \breve{F}, \widetilde{F}, F_{*}\right): \mathcal{A} \rightarrow \mathcal{A}^{\prime}$ is homotopic to a braided Ann-functor $F^{\prime}=\left(F^{\prime}, \breve{F}^{\prime}, \widetilde{F}^{\prime}, F_{*}^{\prime}\right)$, where $F^{\prime} 1=1^{\prime}$, and $F_{*}^{\prime}=i d_{1^{\prime}}$.

Proof Consider the family of isomorphisms in $\mathcal{A}^{\prime}$ :

$$
\theta_{X}= \begin{cases}i d_{F X} & \text { if } X \neq 1 \\ F_{*}^{-1} & \text { if } X=1,\end{cases}
$$

with $X \in \mathcal{A}$. Then we can construct uniquely a braided Ann-functor $F^{\prime}$ from $F$ such that $\theta: F \rightarrow F^{\prime}$ becomes a homotopy, where

$$
\begin{gathered}
F^{\prime} X=\left\{\begin{array}{ll}
F X & \text { if } X \neq 1, \\
1^{\prime} & \text { if } X=1 ;
\end{array} F^{\prime}(f: X \rightarrow Y)=\left(\theta_{X} F(f)\left(\theta_{Y}\right)^{-1}: F^{\prime} X \rightarrow F^{\prime} Y\right) ;\right. \\
\breve{F}_{X, Y}^{\prime}=\left(\theta_{X} \oplus \theta_{Y}\right) \breve{F}_{X, Y} \theta_{X \oplus Y}^{-1} ; \quad \widetilde{F}_{X, Y}^{\prime}=\left(\theta_{X} \otimes \theta_{Y}\right) \widetilde{F}_{X, Y} \theta_{X Y}^{-1} ; \\
F_{*}^{\prime}=\theta_{1} F_{*}=i d_{1} .
\end{gathered}
$$

According to Lemma 3.7, we refer to $(F, \breve{F}, \widetilde{F})$ as a braided Ann-functor.

Proposition 3.8 In the definition of a braided Ann-functor, the commutativity of the diagram (13.2) follows from the other conditions.

Proof We consider the following diagram:

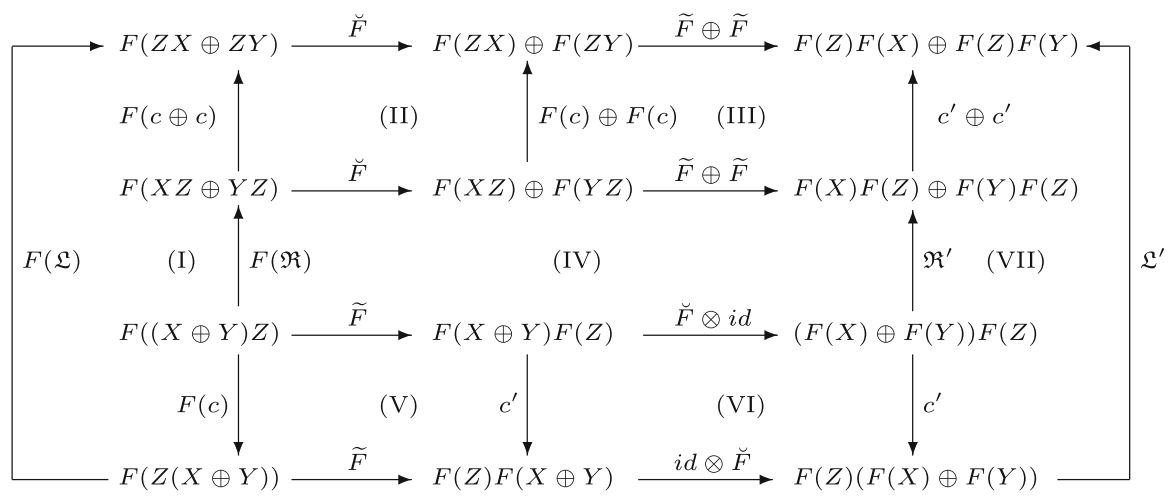


In the above diagram, the regions (I) and (VII) commute thanks to the diagram (12), the region (II) commutes thanks to the naturality of $\breve{F}$, the region (III) commutes thanks to the naturality of $\widetilde{F}$, the region $(\mathrm{V})$ commutes since $(F, \widetilde{F})$ is a braided tensor functor, the region (VI) commutes thanks to the naturality of $c^{\prime}$, the perimeter commutes thanks to the diagram (13.1). Hence, the region (IV) commutes, i.e., the diagram (13.2) commutes.

The structure transport of a monoidal category was presented by Saavedra Rivano [26], Sinh [28]. Later, Quang [20] extended naturally the structure transport to Anncategories. This can be translate to braided Ann-categories.

Proposition 3.9 Let $(F, \breve{F}, \widetilde{F}): \mathcal{A} \rightarrow \mathcal{A}^{\prime}$ be an Ann-equivalence and assume that $\mathcal{A}^{\prime}$ is a braided Ann-category with a braiding $c^{\prime}$. Then $\mathcal{A}$ becomes a braided Anncategory with a braiding $c$ given by the following commutative diagram

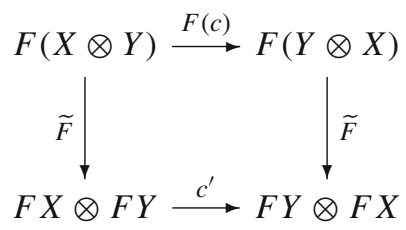

and $(F, \breve{F}, \widetilde{F})$ becomes a braided Ann-equivalence.

Proof We shall prove that $c$ satisfies the diagrams (B1), (B2) and (12).

In order to prove that $c$ satisfies the diagram (B1), we consider the following diagram:

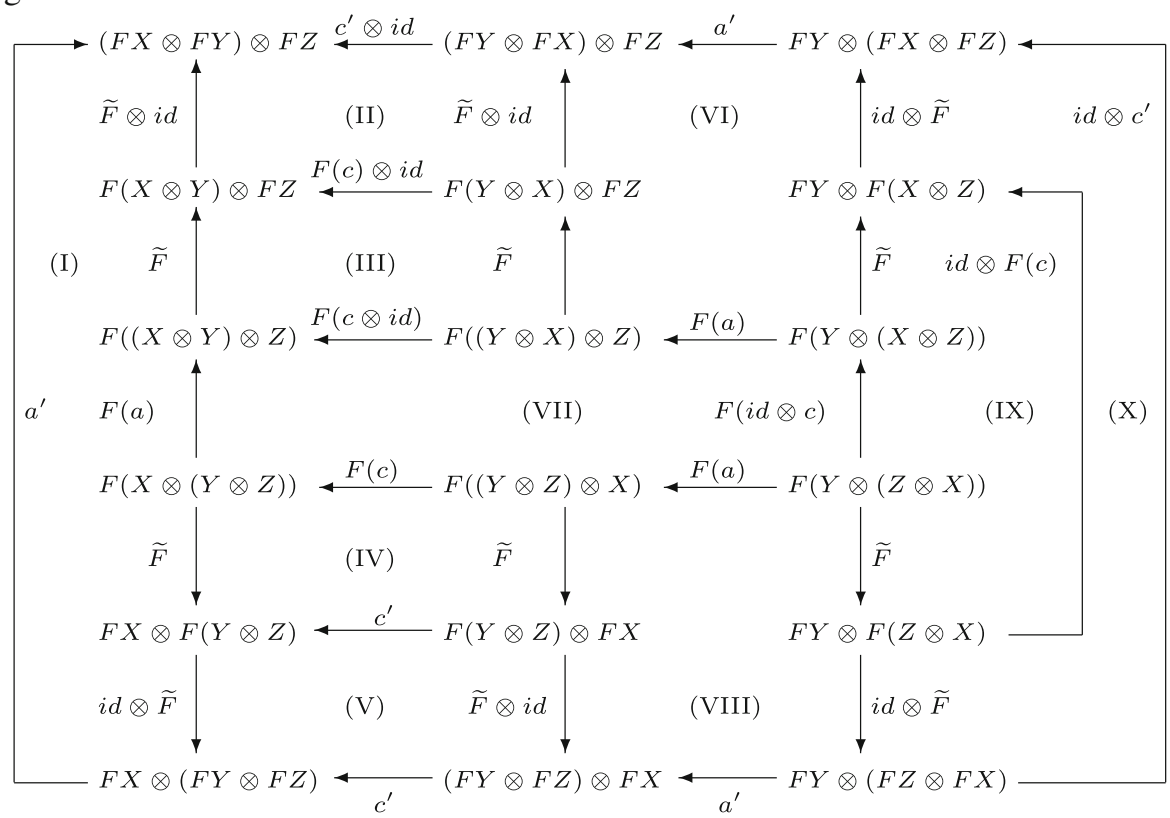


In the above diagram, the regions (I), (VI), (VIII) commute thanks to the condition on the morphism $a$, the regions (II), (IV), (X) commute thanks to the condition on the morphism $c$, the region (III) and (IX) commute thanks to the naturality of $\widetilde{F}$, the region $(\mathrm{V})$ commutes thanks to the naturality of $c^{\prime}$, the perimeter commutes since $\left(\mathcal{A}^{\prime}, \otimes\right)$ is a braided tensor category. Hence, the region (VII) commutes. Since $F$ is an equivalence, the diagram (B1) commutes.

Similarly, the diagram (B2) also commutes.

In order to prove that the diagram (12) commutes, we consider the following diagram. In that diagram, the region (I) commutes thanks to the naturality of $c^{\prime}$, the regions (II) and (VII) commute since $(F, \widetilde{F})$ is a braided $\otimes$-functor, the region (III) commutes thanks to the conditions on $\mathfrak{L}$, the region (V) commutes thanks to the conditions on $\mathfrak{R}$, the region (VI) commutes since $(F, \breve{F})$ is a $\bigoplus$-functor, the perimeter commutes since $\mathcal{A}^{\prime}$ is a braided Ann-category. Hence, the region (IV) commutes. The commutativity of the region (IV) implies the commutativity of the diagram (12) since $F$ is an equivalence.

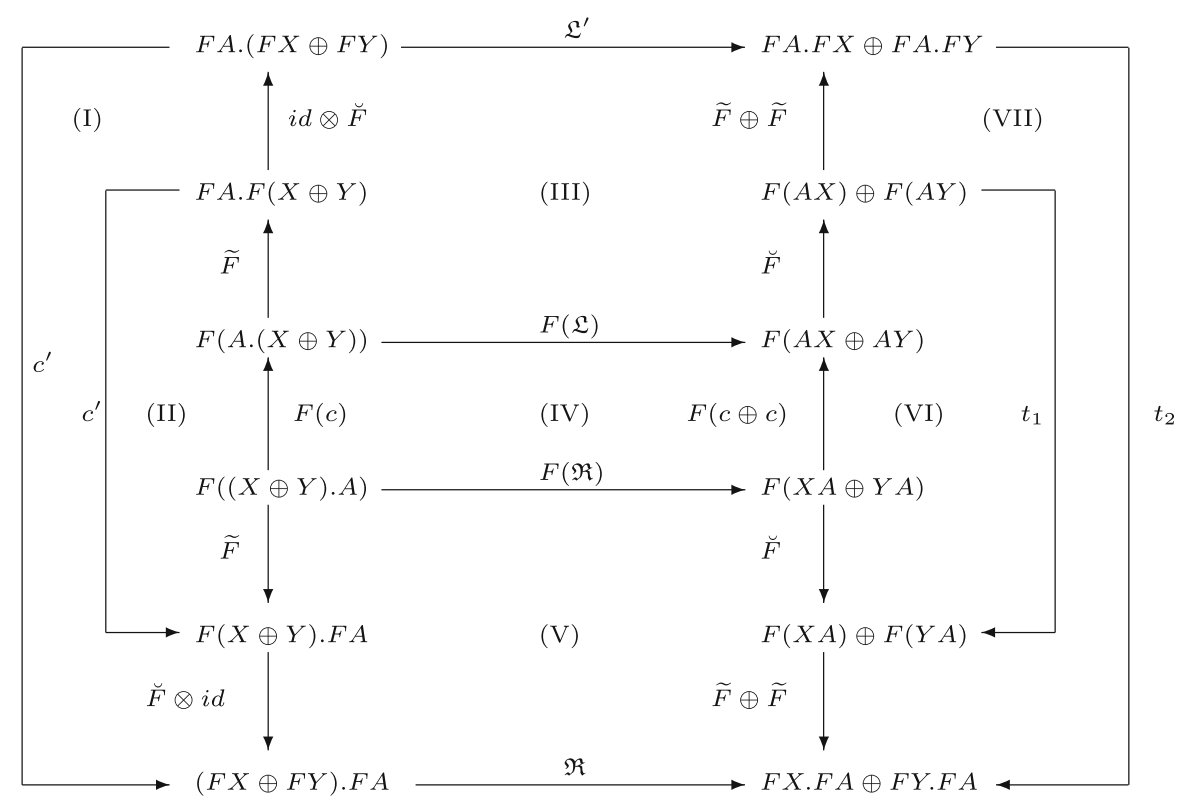

where $t_{1}=F\left(c_{A, X}\right) \oplus F\left(c_{A, Y}\right), t_{2}=c_{F A, F X}^{\prime} \oplus c_{F A, F Y}^{\prime}$.

In Sect. 4, we shall use the structure transport to construct a reduced braided Anncategory of a braided Ann-category.

\section{Reduced braided Ann-categories}

Let $\mathcal{A}$ be a braided $A n n$-category with the family of constraints

$$
\left(a^{+}, c^{+},(0, g, d), a, c,(1, l, r), \mathfrak{L}, \mathfrak{R}\right) .
$$


Then, according to [20], the set $\pi_{0} \mathcal{A}$ of isomorphism classes of objects in $\mathcal{A}$ is a ring with the operations,$+ \times$, induced by the functors $\oplus, \otimes$ on $\mathcal{A}$, and $\pi_{1} \mathcal{A}=\operatorname{Aut}(0)$ is a $\pi_{0} \mathcal{A}$-bimodule with actions: $\lambda_{X}(u), \quad u s=\rho_{X}(u)$,

where $X \in s, s \in \pi_{0} \mathcal{A}, u \in \pi_{1} \mathcal{A}$, and the functions $\lambda, \rho$ are defined as follows:
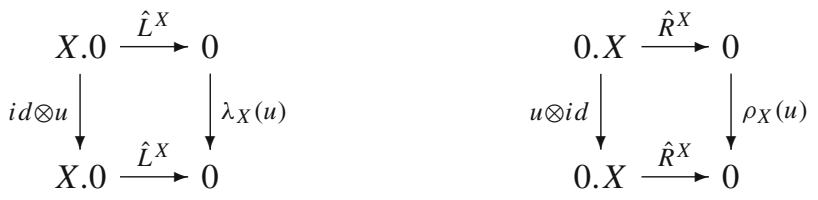

where $\hat{L}^{X}$ (respectively $\left.\hat{R}^{X}\right)$ is the isomorphirm induces by the pair $\left(L^{X}, \breve{L}^{X}\right)$ (respectively $\left(R^{X}, \breve{R}^{X}\right)$ ) (for details, see Proposition 1.3 [21]).

By the braiding property of $\otimes, \pi_{0} \mathcal{A}$ is a commutative ring. Moreover, the two-sided actions of the ring $\pi_{0} \mathcal{A}$ on $\pi_{1} \mathcal{A}$ coincide. This is shown in the following proposition.

Proposition 4.1 In a braided Ann-category $\mathcal{A}, \lambda=\rho$.

Proof It follows directly from Proposition 4.2 [23] and the naturality of the braiding $c$.

Let $\mathcal{A}$ be a braided $A n n$-category. Let $s \mathcal{A}$ be the reduced $A n n$-category of $\mathcal{A}$. Hence, we shall transport the braiding $c$ of $\mathcal{A}$ to $s \mathcal{A}$ to make $s \mathcal{A}$ a braided $A n n$-category.

First, let us recall the main steps of the construction of a reduced Ann-category $s \mathcal{A}$ of an $A n n$-category $\mathcal{A}$, thanks to the structure transport (for details, see [20]). The objects of $s \mathcal{A}$ are the elements of $\pi_{0} \mathcal{A}$, morphisms are automorphisms $(r, a): r \rightarrow$ $r, r \in \pi_{0} \mathcal{A}, a \in \pi_{1} \mathcal{A}$. The composition law of two morphisms is defined by

$$
(r, a) \circ(r, b)=(r, a+b) .
$$

In $\mathcal{A}$, we choose the representatives $X_{s}, s \in \pi_{0} \mathcal{A}$ such that $X_{0}=0, X_{1}=1$, and a family of isomorphisms $i_{X}: X \rightarrow X_{S}$ such that $i_{X_{s}}=i d_{X_{s}}$. Then, we obtain two functors:

$$
\begin{array}{ll}
G: \mathcal{A} \rightarrow s \mathcal{A}, & H: s \mathcal{A} \rightarrow \mathcal{A}, \\
G(X)=[X]=s, & H(s)=X_{s}, \\
G(f)=\left(s, \gamma_{X_{s}}^{-1}\left(i_{Y} f i_{X}^{-1}\right)\right), & H(s, u)=\gamma_{X_{s}}(u),
\end{array}
$$

for $X, Y \in s, f: X \rightarrow Y$, and $\gamma_{X}$ is determined by the following commutative diagram:

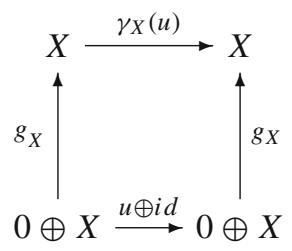


Then, the operations on $s \mathcal{A}$ are given by:

$$
\begin{aligned}
s \oplus t & =G(H(s) \oplus H(t))=s+t, \\
s \otimes t & =G(H(s) \otimes H(t))=s t, \\
(s, u) \oplus(t, v) & =G(H(s, u) \oplus H(t, v))=(s+t, u+v), \\
(s, u) \otimes(t, v) & =G(H(s, u) \otimes H(t, v))=(s t, s v+u t),
\end{aligned}
$$

where $s, t \in \pi_{0} \mathcal{A}, u, v \in \pi_{1} \mathcal{A}$. Obviously, they do not depend on the choice of the representative system $X_{S}, i_{X}$.

Keeping in mind the similar construction done for categorical groups by Sinh in [28], we can provide $s \mathcal{A}$ with the needed constraints using the constraints of $\mathcal{A}$ and a stick (in French, épinglage). A stick in $\mathcal{A}$ consists of a representatives $\left(X_{S}\right)_{s \in \pi_{0} \mathcal{A}}$ such that $X_{0}=0, X_{1}=1$ and isomorphisms

$$
\varphi_{s, t}: X_{s} \oplus X_{t} \rightarrow X_{s+t}, \quad \psi_{s, t}: X_{s} X_{t} \rightarrow X_{s t},
$$

for all $s, t \in \pi_{0} \mathcal{A}$, such that

$$
\begin{array}{ll}
\varphi_{0, t}=g_{X_{t}}, & \varphi_{s, 0}=d_{X_{s}}, \\
\psi_{1, t}=l_{X_{t}}, & \psi_{s, 1}=r_{X_{s}}, \psi_{0, t}=\hat{R}^{X_{t}}, \quad \psi_{s, 0}=\hat{L}^{X_{s}} .
\end{array}
$$

For the two operations $\oplus, \otimes$ of $s \mathcal{A}$, the unit constraints are chosen, respectively, as $(0, i d, i d)$ and $(1, i d, i d)$. Put $\varphi=\left(\varphi_{s, t}\right)$ and $\psi=\left(\psi_{s, t}\right)$, we can determine the constraints $\xi, \eta, \alpha, \lambda, \rho$ which are compatible, respectively, with the constraints $a^{+}, c^{+}, a, \mathfrak{L}, \mathfrak{R}$ of $\mathcal{A}$ via $H, \breve{H}=\varphi^{-1}, \widetilde{H}=\psi^{-1}, H_{*}=i d_{1}$. Then

$$
(\mathcal{S}, \xi, \eta,(0, i d, i d), \alpha,(1, i d, i d), \lambda, \rho)
$$

is an Ann-category which is equivalent to $\mathcal{A}$ by the $A n n$-equivalence $H$. Besides, the functor $G: \mathcal{A} \rightarrow s \mathcal{A}$ together with functor isomorphisms

$$
\breve{G}_{X, Y}=G\left(i_{X} \oplus i_{Y}\right), \quad \widetilde{G}_{X, Y}=G\left(i_{X} \otimes i_{Y}\right), \quad G_{*}=i d_{1},
$$

is an Ann-equivalence, quasi-inverse of $H$.

Proposition 4.2 If $\mathcal{A}$ is a braided Ann-category with the braiding $c$, then $s \mathcal{A}$ is a braided Ann-category with an induced braiding $c^{\prime}=(\bullet, \beta)$ given by the following commutative diagram:

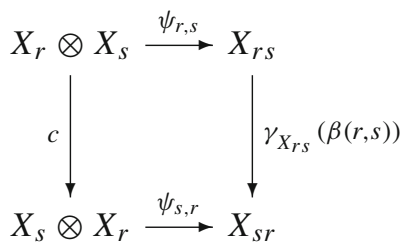

Moreover, $(H, \breve{H}, \widetilde{H})$ and $(G, \breve{G}, \widetilde{G})$ are braided Ann-equivalences. 
Proof It follows directly from Proposition 3.9.

We refer to $s \mathcal{A}$ as the reduced braided Ann-category of $\mathcal{A}$, and $G, H$ as the canonical braided Ann-equivalences. The reduced braided Ann-category $s \mathcal{A}$ of the braided Ann-category $\mathcal{A}$ is called a braided Ann-category of type $(R, M)$ if $\pi_{0} \mathcal{A}$ is $R$ and $\pi_{1} \mathcal{A}$ is $M$.

We now describe in detail the constraints of the braided Ann-category $s \mathcal{A}$ of the type $(R, M)$. We say that the family $(h, \beta)$, where $h=(\xi, \eta, \alpha, \lambda, \rho)$, is a structure of $s \mathcal{A}$.

Proposition 4.3 In a braided Ann-category of type $(R, M)$, the unit constraints are strict, and the other constraints are induced by a structure $(h, \beta) \in Z_{a b}^{3}(R, M)$.

Proof It follows from the axiomatics of a braided Ann-category.

Remark 4.4 If $R$ acts trivially on $M$, the relations $S 13, S 14$ show that $(\alpha, \beta)$ is an abelian 3-cocycle of the additive group $R_{+}$with coefficients in $M$, that is, $(\alpha, \beta) \in$ $H_{a b}^{3}\left(R_{+}, M\right)$ in the sense of Eilenberg-Mac Lane [15].

We now consider the independence of induced constraints of $s \mathcal{A}$ if the stick changes.

Proposition 4.5 If $s \mathcal{A}$ and $s^{\prime} \mathcal{A}$ are two reduced braided Ann-categories of a braided Ann-category $\mathcal{A}$, with, respectively, two sticks $\left(X_{s}, \varphi, \psi\right),\left(X_{s}^{\prime}, \varphi^{\prime}, \psi^{\prime}\right)$, then:

(i) There exists a braided Ann-equivalence $(F, \breve{F}, \widetilde{F}): s^{\prime} \mathcal{A} \rightarrow s \mathcal{A}$, where $F=i d$.

(ii) Two structures $(h, \beta)$ and $\left(h^{\prime}, \beta^{\prime}\right)$ inducing, respectively, the constraints of $s \mathcal{A}$ and $s^{\prime} \mathcal{A}$ belong to the same cohomology class of the commutative ring $R$ with coefficients in $R$-module $M$.

Proof (i) Let $G: \mathcal{A} \rightarrow s \mathcal{A}$ and $H^{\prime}: s^{\prime} \mathcal{A} \rightarrow \mathcal{A}$ be canonical braided Annequivalences corresponding to $\left(X_{s}, \varphi, \psi\right),\left(X_{s}^{\prime}, \varphi^{\prime}, \psi^{\prime}\right)$. The composition $F=$ $G H^{\prime}: s^{\prime} \mathcal{A} \rightarrow s \mathcal{A}$ is a braided $A n n$-equivalence. $s \mathcal{A}$ and $s^{\prime} \mathcal{A}$ have the same underlying categories, and it is easy to see that $F=i d_{s \mathcal{A}}$ is a functor.

(ii) Put $\mu=\widetilde{G H^{\prime}}, v=\widetilde{G H^{\prime}}$. Then, from the compatibility of the braided Annfunctor $(F=i d, \mu, v)$ with, respectively, the constraints of $s \mathcal{A}$ and $s^{\prime} \mathcal{A}$, we deduce the required relations.

Clearly, we have

Corollary 4.6 Two structures $(h, \beta),\left(h^{\prime}, \beta^{\prime}\right)$ are cohomologous iff there are two families of constraints of a braided Ann-category of type $(R, M)$ compatible with each other by the braided Ann-equivalence $(F, \breve{F}, \widetilde{F})$, where $F=i d_{\mathcal{A}}$.

We call a trace of the structure $(h, \beta)$ the map

$$
R \rightarrow M, \quad x \mapsto \beta(x, x)
$$

Corollary 4.7 If $(h, \beta)$ and $\left(h^{\prime}, \beta^{\prime}\right)$ are two cohomologous structures, then $[h]=\left[h^{\prime}\right] \in H_{\text {MacL }}^{3}(R, M)$ and the braidings $\beta, \beta^{\prime}$ have the same trace.

In Sect. 6, we present the third invariant of a braided Ann-category. 


\section{Classification of braided Ann-functors of type $(p, q)$}

We now prove that each braided Ann-functor $(F, \breve{F}, \widetilde{F}): \mathcal{A} \rightarrow \mathcal{A}^{\prime}$ induces a braided $A n n$-functor $s F$ of the reduced braided Ann-categories. Throughout this section, let $\mathcal{S}, \mathcal{S}^{\prime}$ be, respectively, braided Ann-categories of the form $(R, M, h, \beta)$, $\left(R^{\prime}, M^{\prime}, h^{\prime}, \beta^{\prime}\right)$.

Definition 5.1 A functor $F: \mathcal{S} \rightarrow \mathcal{S}^{\prime}$ is called a functor of type $(p, q)$ if

$$
F(x)=p(x), \quad F(x, a)=(p(x), q(a)),
$$

where $p: R \rightarrow R^{\prime}$ is a ring homomorphism and $q: M \rightarrow M^{\prime}$ is a group homomorphism satisfying

$$
q(x a)=p(x) q(a), \quad x \in R, a \in M .
$$

$M^{\prime}$ can be regarded as a $R$-module with action $s a^{\prime}=p(s) a^{\prime}$, then $q$ is a $R$-module homomorphism. We say that $(p, q)$ is a pair of homomorphisms and call the function

$$
k=q_{*}(h, \beta)-p^{*}\left(h^{\prime}, \beta^{\prime}\right),
$$

where $p^{*}, q_{*}$ are canonical homomorphisms

$$
Z_{a b}^{3}(R, M) \stackrel{q_{*}}{\longrightarrow} Z_{a b}^{3}\left(R, M^{\prime}\right) \stackrel{p^{*}}{\longleftarrow} Z_{a b}^{3}\left(R^{\prime}, M^{\prime}\right),
$$

an obstruction of the functor of type $(p, q)$.

Proposition 5.2 Each braided Ann-functor from $\mathcal{S}$ to $\mathcal{S}^{\prime}$ is a functor of type $(p, q)$.

Proof Clearly, each braided Ann-functor is of course an Ann-functor. By Proposition 4.3 [22], the proof is completed.

Proposition 5.3 Let $\mathcal{A}$ and $\mathcal{A}^{\prime}$ be two braided Ann-categories. Then each braided Ann-functor $\left(F, \breve{F}, \widetilde{F}, F_{*}\right): \mathcal{A} \rightarrow \mathcal{A}^{\prime}$ induces a braided Ann-functor $s F: s \mathcal{A} \rightarrow$ $s \mathcal{A}^{\prime}$ of type $(p, q)$, where

$$
\begin{aligned}
& p=\pi_{0}(F): \pi_{0} \mathcal{A} \rightarrow \pi_{0} \mathcal{A}^{\prime} ;[X] \mapsto[F X], \\
& q=\pi_{1}(F): \pi_{1} \mathcal{A} \rightarrow \pi_{1} \mathcal{A}^{\prime} ; \quad u \mapsto \gamma_{F 0}^{-1}(F u),
\end{aligned}
$$

in which $\gamma$ is the map determined by the diagram (15) having the following properties:

(i) $F$ is an equivalence iff $\pi_{0}(F), \pi_{1}(F)$ are isomorphisms.

(ii) The braided Ann-functor $s$ satisfies the following commutative diagram: 


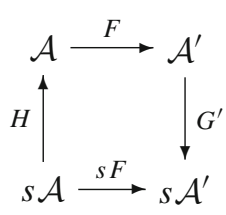

where $H, G^{\prime}$ are canonical braided Ann-equivalences.

Proof Set $s F=G^{\prime} \circ F \circ H$. Since $F, H, G^{\prime}$ are braided $A n n$-functors, so is $s F$.

(i) One can verify that (see Theorem 4.6 [20] for details) $s F$ is of type $\left(\pi_{0}(F), \pi_{1}(F)\right)$.

(ii) According to Proposition 4.2 [22], we infer that the functor $s F$ makes the diagram (16) commute.

Remark 5.4 If $F=\left(F, \breve{F}, \widetilde{F}, F_{*}\right): \mathcal{A} \rightarrow \mathcal{A}^{\prime}$ is a braided $A n n$-functor, the induced braided $A n n$-functor $s F: s \mathcal{A} \rightarrow s \mathcal{A}^{\prime}$ satisfies $s F(1)=1^{\prime}$ and $(s F)_{*}=\left(1^{\prime}, \gamma_{1^{\prime}}^{-1}\left(F_{*} \circ\right.\right.$ $\left.i_{F 1}^{-1}\right)$ ). Moreover, if $F 1=1^{\prime}$ and $F_{*}=i d_{1^{\prime}}$, then $(s F)_{*}=i d_{1^{\prime}}$.

If $F:(R, A, h) \rightarrow\left(R^{\prime}, A^{\prime}, h^{\prime}\right)$ is a braided Ann-functor, then

$$
\breve{F}_{x, y}=(F(x+y), \mu(x, y)), \widetilde{F}_{x, y}=(F(x y), v(x, y)), x, y \in R
$$

and $g_{F}=(\mu, v) \in Z_{S h a b}^{2}$. We say that the pair of functions $g_{F}=(\mu, v)$ associated to $(\breve{F}, \widetilde{F})$, and $F$ can be regarded as a triple $\left(p, q, g_{F}\right)$. Thanks to the compatibility of $F$ with the constraints, we have

$$
q_{*}(h, \beta)-p^{*}\left(h^{\prime}, \beta^{\prime}\right)=\partial\left(g_{F}\right),
$$

Moreover, two braided Ann-functors $\left(F, g_{F}\right),\left(F^{\prime}, g_{F^{\prime}}\right)$ are homotopic iff $F^{\prime}=F$, it means that they are of the same type $(p, q)$, and there exists a map $t: R \rightarrow M^{\prime}$, such that

$$
g_{F^{\prime}}=g_{F}+\partial_{a b} t
$$

Denote by

$$
\operatorname{Hom}_{(p, q)}^{B r A n n}\left[\mathcal{S}, \mathcal{S}^{\prime}\right]
$$

the set of homotopy classes of braided Ann-functors of type $(p, q)$ from $\mathcal{S}$ to $\mathcal{S}^{\prime}$.

Theorem 5.5 The functor $F: \mathcal{S} \rightarrow \mathcal{S}^{\prime}$ of type $(p, q)$ is a braided Ann-functor iff its obstruction $[k]=0$ in $H_{a b}^{3}\left(R, M^{\prime}\right)$. Then, there exist bijections:

$$
\begin{gathered}
\operatorname{Hom}_{(p, q)}^{B r A n n}\left[\mathcal{S}, \mathcal{S}^{\prime}\right] \leftrightarrow H_{a b}^{2}\left(R, M^{\prime}\right), \\
\operatorname{Aut}(F) \leftrightarrow Z_{a b}^{1}\left(R, M^{\prime}\right) .
\end{gathered}
$$


Proof Let $F: \mathcal{S} \rightarrow \mathcal{S}^{\prime}$ be a braided Ann-functor of type $(p, q)$. Then, by the equation (18), the obstruction of $F$ vanishes in the group $H_{a b}^{3}\left(R, M^{\prime}\right)$.

Conversely, if the obstruction of the functor $F$ vanishes in the group $H_{a b}^{3}\left(R, M^{\prime}\right)$, then there exists a 2-cochain $g=(\mu, v)$ such that $k=\partial g$. This means

$$
q_{*}(h, \beta)-p^{*}\left(h^{\prime}, \beta^{\prime}\right)=\partial g .
$$

Take $\breve{F}, \widetilde{F}$ be functor morphisms associated to the maps $\mu$, v by (17). Then, $(F, \breve{F}, \widetilde{F})$ is a braided Ann-functor.

- According to Theorem 4.5 [22], we have a bijection

$$
\Phi: \operatorname{Hom}_{(p, q)}^{A n n}\left[\mathcal{S}, \mathcal{S}^{\prime}\right] \rightarrow H_{M a c L}^{2}\left(R, M^{\prime}\right)
$$

The bijection $\Phi$ is defined as follows (for details, see Theorem 4.5 [22]). For an Ann-functor $(F, \breve{F}, \widetilde{F}): \mathcal{S} \rightarrow \mathcal{S}^{\prime}$, we have

$$
q_{*} h-p^{*} h^{\prime}=\partial_{M a c L} g_{F} .
$$

Let $g_{F}$ be fixed. If $(K, \breve{K}, \widetilde{K}): \mathcal{S} \rightarrow \mathcal{S}^{\prime}$ is a Ann-functor of type $(p, q)$, then

$$
q_{*} h-p^{*} h^{\prime}=\partial_{M a c L} g_{K} .
$$

Hence, $g_{F}-g_{K}$ is a 2-cocycle. The map $\Phi$ is given by:

$$
\Phi([K])=\left[g_{F}-g_{K}\right] .
$$

Now, for braided Ann-functors $F, K$, the functions $g_{F}, g_{K}$ are 2-cochains of the commutative ring $R$ with coefficients in the $R$-module $M$ and $g_{F}-g_{K} \in Z_{a b}^{2}\left(R, M^{\prime}\right)$. Besides, since $Z_{a b}^{2}\left(R, M^{\prime}\right) \subset Z_{M a c L}^{2}\left(R, M^{\prime}\right)$ and $B_{a b}^{2}\left(R, M^{\prime}\right)=B_{M a c L}^{2}\left(R, M^{\prime}\right)$, we have

$$
H_{a b}^{2}\left(R, M^{\prime}\right) \subset H_{M a c L}^{2}\left(R, M^{\prime}\right)
$$

Let $\Phi^{\prime}$ be the restriction of the map $\Phi$ on the set $\operatorname{Hom}_{(p, q)}^{B r A n n}\left[\mathcal{S}, \mathcal{S}^{\prime}\right]$, we obtain an injection

$$
\Phi^{\prime}: \operatorname{Hom}_{(p, q)}^{B r A n n}\left[\mathcal{S}, \mathcal{S}^{\prime}\right] \rightarrow H_{a b}^{2}\left(R, M^{\prime}\right)
$$

Now, if $g$ is an arbitrary 2-cocycle in $Z_{a b}^{2}\left(R, M^{\prime}\right)$, we have

$$
\partial\left(g_{F}-g\right)=\partial g_{F}-\partial g=\partial g_{F}=q_{*}(h, \beta)-p^{*}\left(h^{\prime}, \beta^{\prime}\right) .
$$

Thus, there exists a braided Ann-functor $(K, \breve{K}, \widetilde{K}): \mathcal{S} \rightarrow \mathcal{S}^{\prime}$ of type $(p, q)$, where the isomorphisms $\breve{K}, \widetilde{K}$ are associated with the 2-cochain $g_{F}-g$. Clearly, $\Phi^{\prime}([K])=$ $[g]$, which means that $\Phi^{\prime}$ is a surjection. Thus, $\Phi^{\prime}$ is a bijection. 
- In the equation (19), with $F^{\prime}=F$, we infer $\partial t=0$, i.e., $t \in Z_{a b}^{1}\left(R, M^{\prime}\right)$. Thus, there exists a map

$$
\begin{aligned}
\operatorname{Aut}(F) & \rightarrow Z_{a b}^{1}\left(R, M^{\prime}\right), \\
u & \mapsto t .
\end{aligned}
$$

It is easy to see that the above map is a bijection.

\section{Classification Theorems}

We refer to BrAnn as the category whose objects are braided Ann-categories, and whose morphisms are braided Ann-functors between braided Ann-categories.

Similarly to the Classification Theorem for graded Picard categories (Theorem 3.12 [5]), we determine $\mathbf{H}_{\mathrm{BrAnn}}^{\mathbf{3}}$ to be the category whose objects are the triples $(R, M,[h, \beta])$, where $[h, \beta] \in H_{a b}^{3}(R, M)$ and $(R, M, h, \beta)$ is a braided Anncategory. A morphism $(p, q):(R, M,[h, \beta]) \rightarrow\left(R^{\prime}, M^{\prime},\left[h^{\prime}, \beta^{\prime}\right]\right)$ of $\mathbf{H}_{\mathbf{B r A n n}}^{\mathbf{3}}$ is a pair $(p, q)$ such that there exists $g=(\mu, v): R^{2} \rightarrow M^{\prime}$ and $(p, q, g)$ is a braided Ann-functor $(R, M, h, \beta) \rightarrow\left(R^{\prime}, M^{\prime}, h^{\prime}, \beta^{\prime}\right)$, i.e., $\left[p^{*}\left(h^{\prime}, \beta^{\prime}\right)\right]=\left[q_{*}(h, \beta)\right] \in$ $H_{a b}^{3}\left(R, M^{\prime}\right)$. The composition law in $\mathbf{H}_{\mathbf{B r A n n}}^{\mathbf{3}}$ is defined by

$$
\left(p^{\prime}, q^{\prime}\right) \circ(p, q)=\left(p^{\prime} p, q^{\prime} q\right)
$$

Note that, two braided Ann-functors $F, F^{\prime}: \mathcal{A} \rightarrow \mathcal{A}^{\prime}$ are homotopic iff $\pi_{i}(F)=$ $\pi_{i}\left(F^{\prime}\right), i=0,1$ and $\left[g_{F}\right]=\left[g_{F^{\prime}}\right]$. Denoting by

$$
\operatorname{Hom}_{(p, q)}^{B r A n n}\left[\mathcal{A}, \mathcal{A}^{\prime}\right]
$$

the set of homotopy classes of braided $A n n$-functors $\mathcal{A} \rightarrow \mathcal{A}^{\prime}$ which induce the same pair $(p, q)$, we state the main theorem of this section.

Theorem 6.1 (The classification theorem) There exists a functor

$$
\begin{aligned}
d: \text { BrAnn } & \rightarrow \mathbf{H}_{\text {BrAnn }}^{\mathbf{3}}, \\
\mathcal{A} & \mapsto\left(\pi_{0} \mathcal{A}, \pi_{1} \mathcal{A},\left[(h, \beta)_{\mathcal{A}}\right]\right), \\
F=(F, \breve{F}, \widetilde{F}) & \mapsto\left(\pi_{0}(F), \pi_{1}(F)\right),
\end{aligned}
$$

with the following properties:

(i) $d F$ is an isomorphism iff $F$ is an equivalence;

(ii) $d$ is a surjection over the set of objects;

(iii) $d$ is full but not faithful. Indeed, for any arrow $(p, q): d \mathcal{A} \rightarrow d \mathcal{A}^{\prime}$ in $\mathbf{H}_{\mathbf{B r A n n}}^{\mathbf{3}}$, there is a bijection:

$$
d: \operatorname{Hom}_{(p, q)}^{B r A n n}\left[\mathcal{A}, \mathcal{A}^{\prime}\right] \rightarrow H_{a b}^{2}\left(\pi_{0} \mathcal{A}, \pi_{1} \mathcal{A}^{\prime}\right)
$$


Proof In the braided $A n n$-category $\mathcal{A}$, for any stick $\left(X_{S}, i_{X}\right)$, we can build a reduced braided $A n n$-category $\left(\pi_{0} \mathcal{A}, \pi_{1} \mathcal{A}, h, \beta\right)$. When the choice of stick is changed, the 3 -cocycle $(h, \beta)$ is replaced by $\left(h^{\prime}, \beta^{\prime}\right)$ which is cohomologous with $(h, \beta)$. So $\mathcal{A}$ defines uniquely an element $[(h, \beta)] \in H_{a b}^{3}\left(\pi_{0} \mathcal{A}, \pi_{1} \mathcal{A}\right)$. It means that $d$ is a map on objects.

For the braided Ann-functors

$$
\mathcal{A} \stackrel{F}{\longrightarrow} \mathcal{A}^{\prime} \stackrel{F^{\prime}}{\longrightarrow} \mathcal{A}^{\prime \prime}
$$

it is easy to see that $d\left(F^{\prime} \circ F\right)=\left(d F^{\prime}\right) \circ(d F)$, and $d\left(i d_{\mathcal{A}}\right)=i d_{d \mathcal{A}}$. Thus $d$ is a functor.

(i) Thanks to Proposition 5.3.

(ii) If $(R, M,[h, \beta])$ is an object of $\mathbf{H}_{\mathrm{BrAnn}}^{\mathbf{3}}$, then $\mathcal{S}=(R, M, h, \beta)$ is a braided Ann-category of type $(R, M)$ and obviously, $d \mathcal{S}=(R, M,[h, \beta])$.

(iii) Assume that $(p, q)$ is a morphism in $\operatorname{Hom}_{\mathbf{H}_{\mathrm{BrAnn}}^{3}}\left(d \mathcal{A}, d \mathcal{A}^{\prime}\right)$. Then, there exists a function $g=(\mu, v), \mu, v:\left(\pi_{0} \mathcal{A}\right)^{2} \rightarrow \pi_{1} \mathcal{A}^{\prime}$ such that

$$
q_{*}(h, \beta)_{\mathcal{A}}=p^{*}(h, \beta)_{\mathcal{A}^{\prime}}+\partial g .
$$

Therefore,

$$
K=(p, q, g):\left(\pi_{0} \mathcal{A}, \pi_{1} \mathcal{A},(h, \beta)_{\mathcal{A}}\right) \rightarrow\left(\pi_{0} \mathcal{A}^{\prime}, \pi_{1} \mathcal{A}^{\prime},(h, \beta)_{\mathcal{A}^{\prime}}\right)
$$

is a braided Ann-functor. Then, according to Proposition 5.3, the composition of braided $A n n$-functors $F=H^{\prime} \circ K \circ G: \mathcal{A} \rightarrow \mathcal{A}^{\prime}$ induces a braided $A n n$-functor $s F$. It is ea*sy to see that $d F=(p, q)$. This means that the functor $d$ is full.

To show that (21) is a bijection, we prove that the map

$$
\begin{aligned}
s: \operatorname{Hom}_{(p, q)}^{B r A n n}\left[\mathcal{A}, \mathcal{A}^{\prime}\right] & \rightarrow \operatorname{Hom}_{(p, q)}^{B r A n n}\left[s \mathcal{A}, s \mathcal{A}^{\prime}\right], \\
{[F] } & \mapsto[s F]
\end{aligned}
$$

is a bijection.

Clearly, if $F, F^{\prime}: \mathcal{A} \rightarrow \mathcal{A}^{\prime}$ are homotopic, then the reduced braided Ann-functors $s F, s F^{\prime}$ are homotopic. Conversely, if $s F, s F^{\prime}$ are homotopic, then the compositions $E=H^{\prime}(s F) G$ and $E^{\prime}=H^{\prime}\left(s F^{\prime}\right) G$ are homotopic. The braided Ann-functors $E, E^{\prime}$ are homotopic to, respectively, $F, F^{\prime}$. So, $F$ and $F^{\prime}$ are homotopic, i.e., $s$ is injective.

Now, if $K=(p, q, g): s \mathcal{A} \rightarrow s \mathcal{A}^{\prime}$ is a braided $A n n$-functor, then the composition

$$
F=H^{\prime} \circ K \circ G: \mathcal{A} \rightarrow \mathcal{A}^{\prime}
$$

is a braided $A n n$-functor such that $s F=K$, i.e., $s$ is a surjection. Now, the bijection (21) is just the composition of (22) and (20). 
According to Proposition 5.3, we may simplify the problem of equivalence classification of braided Ann-categories by the classification of braided Ann-categories which have the same (up to an isomorphism) first two invariants.

Let $R$ be a commutative ring with unit, $M$ be a $R$-module (regarded as a ring with the null multiplication). We say that a braided Ann-category $\mathcal{A}$ has a pre-stick of type $(R, M)$ if there exists a pair of ring isomorphisms $\epsilon=(p, q)$,

$$
p: R \rightarrow \pi_{0} \mathcal{A}, \quad q: M \rightarrow \pi_{1} \mathcal{A},
$$

which is compatible with the module action, i.e.,

$$
q(s u)=p(s) q(u)
$$

for $s \in R, u \in M$. The pair $(p, q)$ is called a pre-stick of type $(R, M)$ of the braided Ann-category $\mathcal{A}$.

A morphism between two braided $A n n$-categories $\mathcal{A}, \mathcal{A}^{\prime}$ whose pre-sticks (respectively, $\left.\epsilon=(p, q), \epsilon^{\prime}=\left(p^{\prime}, q^{\prime}\right)\right)$ are of type $(R, M)$ is a braided Ann-functor $(F, \breve{F}, \widetilde{F}): \mathcal{A} \rightarrow \mathcal{A}^{\prime}$ such that the following diagrams
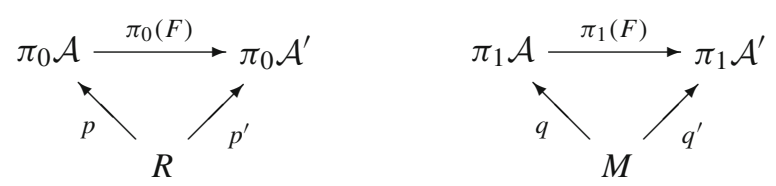

commute.

Clearly, from the definition, we infer that $\pi_{0}(F), \pi_{1}(F)$ are isomorphisms and thus $F$ is an equivalence.

Denote by

\section{$\operatorname{BrAnn}[R, M]$}

the set of iso-classes of braided Ann-categories whose pre-sticks are of type $(R, M)$. Using Proposition 4.5, we can prove the following theorem.

Theorem 6.2 There exists a bijection

$$
\begin{aligned}
\Gamma: \operatorname{BrAnn}[R, M] & \rightarrow H_{a b}^{3}(R, M), \\
{[\mathcal{A}] } & \mapsto q_{*}^{-1} p^{*}\left[(h, \beta)_{\mathcal{A}}\right] .
\end{aligned}
$$

Under this correspondence regular braided Ann-categories corresponds to elements of the group $H_{\text {Shab }}^{3}(R, M)$.

Proof According to Proposition 4.5, each braided Ann-category $\mathcal{A}$ defines uniquely an element $\left[(h, \beta)_{\mathcal{A}}\right] \in H_{a b}^{3}\left(\pi_{0} \mathcal{A}, \pi_{1} \mathcal{A}\right)$, and so it defines an element

$$
\epsilon\left[(h, \beta)_{\mathcal{A}}\right]=q_{*}^{-1} p^{*}\left[(h, \beta)_{\mathcal{A}}\right] \in H_{a b}^{3}(R, M) .
$$


Now, if $F: \mathcal{A} \rightarrow \mathcal{A}^{\prime}$ is a morphism of two braided Ann-categories and $F$ is of pre-stick type $(p, q)$, the induced braided Ann-functor $s F=\left(p, q, g_{F}\right)$, satisfies the equation (18), and so

$$
p^{*}\left[(h, \beta)_{\mathcal{A}^{\prime}}\right]=q_{*}\left[(h, \beta)_{\mathcal{A}^{\prime}}\right]
$$

This implies

$$
\epsilon^{\prime}\left[(h, \beta)_{\mathcal{A}^{\prime}}\right]=\epsilon\left[(h, \beta)_{\mathcal{A}}\right] .
$$

Thus, $\Gamma$ is a map. Moreover, it is injective. Indeed, if $\Gamma(\mathcal{A})=\Gamma\left(\mathcal{A}^{\prime}\right)$, then

$$
\epsilon(h, \beta)_{\mathcal{A}}-\epsilon^{\prime}(h, \beta)_{\mathcal{A}^{\prime}}=\partial g .
$$

So, there exists a braided Ann-functor $J$ of type (id,id) from $\mathcal{I}=\left(R, M, \epsilon(h, \beta)_{\mathcal{A}}\right)$ to $\mathcal{I}^{\prime}=\left(R, M, \epsilon^{\prime}(h, \beta)_{\mathcal{A}^{\prime}}\right)$. The composition

$$
\mathcal{A} \stackrel{G}{\longrightarrow} s \mathcal{A} \stackrel{\epsilon^{-1}}{\longrightarrow} \mathcal{I} \stackrel{J}{\longrightarrow} \mathcal{I}^{\prime} \stackrel{\epsilon^{\prime}}{\longrightarrow} s \mathcal{A}^{\prime} \stackrel{H^{\prime}}{\longrightarrow} \mathcal{A}^{\prime}
$$

shows that $[\mathcal{A}]=\left[\mathcal{A}^{\prime}\right]$, and $\Gamma$ is injective. Obviously, $\Gamma$ is a surjection.

In connection with Harrison cohomology [8], we obtain the following result.

Corollary 6.3 There exists an injection from the Harrison cohomology group $H_{H a r}^{3}(R, M)$ to the set of iso-classes of the braided Ann-categories whose pre-sticks are of type $(R, M)$.

Proof If $\alpha$ is a Harrison 3-cocycle of the commutative ring $R$, with coefficients in $M$, then $\alpha$ together with $\xi=0, \eta=0, \beta=0, \lambda=0, \rho=0$ is a structure of the braided Ann-category $s(R, M)$. Moreover, if $\alpha^{\prime}$ is another Harrison 3-cocycle, then $\alpha^{\prime}-\alpha=\partial(g)$ iff the braided Ann-categories $s(R, M, \alpha)$ and $s\left(R, M, \alpha^{\prime}\right)$ are equivalent.

Acknowledgments The authors are much indebted to the referee and Walter Tholen, whose useful observations greatly improved our exposition.

\section{References}

1. Barr, M.: Cohomology and obstructions: commutative algebras. In: Lecture Notes in Mathematics, vol. 80, pp. 357-375. Springer, Berlin (1969)

2. Bénabou, J.: Catégories avec multiplication. C. R. Acad. Sci. Paris 253, 1887-1890 (1963)

3. Calvo, M., Cegarra, A.M., Quang, N.T.: Higher cohomologies of modules. Algebr. Geometr. Topol. 12, 343-413 (2012)

4. Cegarra, A.M., Khmaladze, E.: Homotopy classification of braided graded categorical groups. J. Pure Appl. Algebra 209, 411-437 (2007)

5. Cegarra, A.M., Khmaladze, E.: Homotopy classification of graded Picard categories. Adv. Math. 213(2), 644-686 (2007)

6. Fröhlich, A., Wall, C.T.C.: Graded monoidal categories. Composit. Math. 28(3), 229-285 (1974) 
7. Garzón, A.R., del Río, A.: Equivariant extensions of categorical groups. Appl. Categor. Struct. 13, 131-140 (2005)

8. Harrison, D.K.: Commutative algebars and cohomology. Trans. Am. Math. Soc 104, 191-204 (1962)

9. Huang, F., Chen, S.H., Chen, W., Zheng, Z.J.: 2-Modules and the Representation of 2-Rings, arXiv: 1005.2831v1 [math.CT] 17 May 2010

10. Joyal, A., Street, R.: Braided tensor categories. Adv. Math. 102, 20-78 (1993)

11. Jibladze, M., Pirashvili, T.: Third Mac Lane cohomology via categorical rings. J. Homotopy Relat. Struct. 2(2), 187-216 (2007)

12. Kapranov, M.M., Voevodsky, V.A.: 2-categories and Zamolodchikov tetrahedra equations. In: Proceedings of Symposia in Pure Mathematics, vol. 56, Part 2 (1994)

13. Laplaza, M.L.: Coherence for distributivity. Lect. Notes Math. 281, 29-65 (1972)

14. Laplaza, M.L.: Coherence for categories with group structure: an alterlative approach. J. Algebra 84, 305-323 (1983)

15. Mac Lane, S.: Cohomology theory of abelian groups. Proc. Int. Congr. Math. 2, 8-14 (1950)

16. Mac Lane, S.: Extensions and obstructions for rings. Ill. J. Math. 2, 316-345 (1958)

17. Mac Lane, S.: Natural associativity and commutativity. Rice. Univ. Stud. 49, 28-46 (1963)

18. Phung, C.T.K., Quang, N.T., Thuy, N.T.: Relation between Ann-categories and ring categories. Commun. Korean Math. Soc. 25(4), 523-535 (2010)

19. Quang, N.T.: Introduction to Ann-categories. Vietnam J. Math. 15(4), 14-24(1987). [arXiv: 0702588v2 [math.CT] 21 Feb 2007]

20. Quang, N.T.: Cohomological classification of Ann-categories. Math. Commun. 18, 151-169 (2013)

21. Quang, N.T.: Structure of Ann-categories and Mac Lane-Shukla cohomology. East-West J. Math. 5(1), 51-66 (2003)

22. Quang, N.T., Hanh, D.D.: Cohomological classification of Ann-functors. East-West J. Math. 11(2), 195-210 (2009)

23. Quang, N.T., Hanh, D.D.: On the braiding of an Ann-category. Asian-Eur. J. Math. 3(4), 647-666 (2010)

24. Quang, N.T., Hanh, D.D., Thuy, N.T.: On the axiomatics of Ann-categories. JP J. Algebra Number Theory Appl. 11(1), 59-72 (2008)

25. Quillen, D.: On the (co)-homology of commutative rings, pp. 65-87. Proc. Symp. Pure Math. Soc, Providence (1970)

26. Saavedra Rivano, N.: Catégories Tannakiennes. In: Lecture Notes in Math, vol. 265. Springer, Berlin (1972)

27. Shukla, U.: Cohomologic des algébres associativé. Ann. Sci. École Norm. Sup. 78, 163-209 (1961)

28. Sinh, H.X.: Gr-catégories, Thèse de doctorat (Université Paris VII, 1975) 